Working Papers of the University of Vaasa,

Department of Mathematics and Statistics,

14

Theorem of completeness for a Dirac-type operator with generalized $\lambda$-depending boundary conditions

Seppo Hassi and Leonid Oridoroga

Preprint, August 2008

University of Vaasa

Department of Mathematics and Statistics

P.O. Box 700, FIN-65101 Vaasa, Finland

Preprints are available at: http://lipas.uwasa.fi/julkaisu/ewp.html 


\title{
THEOREM OF COMPLETENESS FOR A DIRAC-TYPE OPERATOR WITH GENERALIZED $\lambda$-DEPENDING BOUNDARY CONDITIONS
}

\author{
SEPPO HASSI AND LEONID ORIDOROGA
}

\begin{abstract}
A completeness theorem is proved involving a system of integro-differential equations with some $\lambda$-depending boundary conditions. Also some sufficient conditions for the root functions to form a Riesz basis are established.
\end{abstract}

\section{INTRODUCTION}

It is well known [11, Chap. 1, §3] that the system of eigenfunctions and associate functions (SEAF) of the Sturm - Liouville problem

$$
\begin{gathered}
-y^{\prime \prime}+q(x) y=\lambda^{2} y, \\
y^{\prime}(0)-h_{0} y(0)=y^{\prime}(1)-h_{1} y(1)=0,
\end{gathered}
$$

is complete in $L_{2}[0,1]$ for arbitrary complex valued potential $q \in L_{1}[0,1]$ and $h_{0}, h_{1} \in \mathbb{C}$. A similar result is also known for arbitrary nondegenerate boundary conditions (see $[11$, Chap. 1, §3]).

A completeness result for a boundary value problem of arbitrary order differential equations of the form

$$
y^{(n)}+\sum_{j=0}^{n-2} q_{j}(x) y=\lambda^{n} y,
$$

with separated boundary conditions, has been announced by M.V. Keldysh [7] and was first proved by A.A. Shkalikov [17].

In [10] M.M. Malamud and one of the authors have generalized the above mentioned results from [11] to the case of first order systems with arbitrary boundary conditions (not depending on a spectral parameter).

A more specific system, involving $\lambda$-polynomial boundary conditions, is treated in $[20]$ using a linearization method and asymptotic estimates of the Green's function, cf. [12, p. 388]; see also other references in [20].

In [18] and [19] the completeness results for the problem (1.1), (1.2) have been generalized to the case of nonlinear $\lambda$-depending boundary conditions of the form

$$
\left\{\begin{array}{l}
P_{11}(\lambda) y(0)+P_{12}(\lambda) y^{\prime}(0)=0 \\
P_{21}(\lambda) y^{2}\left(\frac{1}{2}\right)+P_{22}(\lambda) y\left(\frac{1}{2}\right) y^{\prime}\left(\frac{1}{2}\right)+P_{23}(\lambda) y^{\prime 2}\left(\frac{1}{2}\right)=0
\end{array}\right.
$$

1991 Mathematics Subject Classification. Primary 34L40; Secondary 47E05.

The present research was supported by the Academy of Finland (project 129092). 
and of the form

$$
\left\{\begin{array}{l}
P_{10}(\lambda) y^{2}(0)+P_{11}(\lambda) y^{\prime 2}(0)+P_{12}(\lambda) y^{2}\left(\frac{1}{3}\right)+P_{13}(\lambda) y^{\prime 2}\left(\frac{1}{3}\right) \\
+P_{14}(\lambda) y(0) y^{\prime}(0)+P_{15}(\lambda) y(0) y\left(\frac{1}{3}\right)+P_{16}(\lambda) y(0) y^{\prime}\left(\frac{1}{3}\right) \\
+P_{17}(\lambda) y^{\prime}(0) y\left(\frac{1}{3}\right)+P_{18}(\lambda) y^{\prime}(0) y^{\prime}\left(\frac{1}{3}\right)+P_{19}(\lambda) y\left(\frac{1}{3}\right) y^{\prime}\left(\frac{1}{3}\right)=0 \\
P_{20}(\lambda) y^{2}(0)+P_{21}(\lambda) y^{\prime 2}(0)+P_{22}(\lambda) y^{2}\left(\frac{1}{3}\right)+P_{23}(\lambda) y^{\prime 2}\left(\frac{1}{3}\right) \\
+P_{24}(\lambda) y(0) y^{\prime}(0)+P_{25}(\lambda) y(0) y\left(\frac{1}{3}\right)+P_{26}(\lambda) y(0) y^{\prime}\left(\frac{1}{3}\right) \\
+P_{27}(\lambda) y^{\prime}(0) y\left(\frac{1}{3}\right)+P_{28}(\lambda) y^{\prime}(0) y^{\prime}\left(\frac{1}{3}\right)+P_{29}(\lambda) y\left(\frac{1}{3}\right) y^{\prime}\left(\frac{1}{3}\right)=0
\end{array}\right.
$$

where $P_{i j}(\lambda)$ are polynomials.

Moreover, in [15] analogous results were obtained for a system with a pair of separated $\lambda$-depending boundary conditions similar to the conditions (1.4) and (1.5).

In the recent papers [21], [22] a related problem concerning the Riesz basis property of the SEAF for a first-order system of the form (1.6) given below with separated boundary conditions, not depending on a spectral parameter, has been established. In the present paper completeness and Riesz basis property of the SEAF are considered for Dirac-type systems with certain $\lambda$-depending boundary conditions. Naturally the result cover the case of Dirac operators, which have been more extensively studied in the literature. In particular, we wish to mention the recent studies on spectral decompositions of 1D periodic Dirac operators and related convergence results by B. Mityagin and P. Djakov; see [13] and [2], [3, Sec. 4].

The paper is organized as follows. In Section 1 we prove a completeness result for the first order systems of certain integro-differential equations involving general linear or quadratic $\lambda$-depending boundary conditions. More precisely, let $B=\operatorname{diag}\left(a^{-1}, b^{-1}\right)$ be a $2 \times 2$ diagonal matrix with $a<0<b$. Consider in $L_{2}[0,1] \oplus L_{2}[0,1]$ a boundary value problem for the first order system of ordinary integro-differential equations of the form

$$
\frac{1}{i} B y^{\prime}+Q(x) y+\int_{0}^{x} M(x, t) y(t) d t=\lambda y .
$$

Here

$$
Q(x, t)=\left(\begin{array}{cc}
0 & q_{1}(x, t) \\
q_{2}(x, t) & 0
\end{array}\right), \quad M(x, t)=\left(\begin{array}{ll}
M_{11}(x, t) & M_{12}(x, t) \\
M_{21}(x, t) & M_{22}(x, t)
\end{array}\right), \quad y(x)=\left(\begin{array}{l}
y_{1}(x) \\
y_{2}(x)
\end{array}\right)
$$

where it is assumed that $q_{j} \in L_{1}[0,1]$ and $M_{i j} \in L_{\infty}(\Omega), \Omega=\{0 \leq t \leq x \leq 1\}, i, j=1,2$. Two types of $\lambda$-depending boundary conditions will be treated. Namely:

(i) arbitrary linear conditions of the form

$$
\left\{\begin{array}{l}
P_{11}(\lambda) y_{1}(0)+P_{12}(\lambda) y_{2}(0)+P_{13}(\lambda) y_{1}(1)+P_{14}(\lambda) y_{2}(1)=0 \\
P_{21}(\lambda) y_{1}(0)+P_{22}(\lambda) y_{2}(0)+P_{23}(\lambda) y_{1}(1)+P_{24}(\lambda) y_{2}(1)=0
\end{array}\right.
$$

and: 
(ii) arbitrary quadratic conditions of the form

$$
\left\{\begin{array}{l}
P_{10}(\lambda) y_{1}^{2}(0)+P_{11}(\lambda) y_{2}^{2}(0)+P_{12}(\lambda) y_{1}^{2}\left(\frac{1}{2}\right)+P_{13}(\lambda) y_{2}^{2}\left(\frac{1}{2}\right) \\
+P_{14}(\lambda) y_{1}(0) y_{2}(0)+P_{15}(\lambda) y_{1}(0) y_{1}\left(\frac{1}{2}\right)+P_{16}(\lambda) y_{1}(0) y_{2}\left(\frac{1}{2}\right) \\
+P_{17}(\lambda) y_{2}(0) y_{1}\left(\frac{1}{2}\right)+P_{18}(\lambda) y_{2}(0) y_{2}\left(\frac{1}{2}\right)+P_{19}(\lambda) y_{1}\left(\frac{1}{2}\right) y_{2}\left(\frac{1}{2}\right)=0 \\
P_{20}(\lambda) y_{1}^{2}(0)+P_{21}(\lambda) y_{2}^{2}(0)+P_{22}(\lambda) y_{1}^{2}\left(\frac{1}{2}\right)+P_{23}(\lambda) y_{2}^{2}\left(\frac{1}{2}\right) \\
+P_{24}(\lambda) y_{1}(0) y_{2}(0)+P_{25}(\lambda) y_{1}(0) y_{1}\left(\frac{1}{2}\right)+P_{26}(\lambda) y_{1}(0) y_{2}\left(\frac{1}{2}\right) \\
+P_{27}(\lambda) y_{2}(0) y_{1}\left(\frac{1}{2}\right)+P_{28}(\lambda) y_{2}(0) y_{2}\left(\frac{1}{2}\right)+P_{29}(\lambda) y_{1}\left(\frac{1}{2}\right) y_{2}\left(\frac{1}{2}\right)=0
\end{array}\right.
$$

where $P_{i j}(\lambda)$ are polynomials.

In Section 2 some general sufficient conditions for polynomials $P_{i j}$ are established in order that the SEAF of the problem (1.6) with separated $\lambda$-depending boundary conditions forms a Riesz basis.

Some of the main results of this paper has been announced without proofs in [5], [6].

\section{TheOrems on COMPleteness of SEAF}

In this section some sufficient conditions for the completeness of the SEAF of the problems (1.6), (1.7) and (1.6), (1.8) in $L^{2}[0,1] \oplus L^{2}[0,1]$ are established. The starting point is to estimate the growth of the solution of the Cauchy problem for the system (1.6) with special initial conditions.

Let

$$
\vec{\varphi}_{\alpha}(x ; \lambda)=\left(\begin{array}{c}
\varphi_{\alpha 1}(x ; \lambda) \\
\varphi_{\alpha 2}(x ; \lambda)
\end{array}\right) \quad \text { and } \quad \vec{\psi}_{\alpha}(x ; \lambda)=\left(\begin{array}{c}
\psi_{\alpha 1}(x ; \lambda) \\
\psi_{\alpha 2}(x ; \lambda)
\end{array}\right)
$$

be the solutions of the Cauchy problem for the system (1.6) with the initial conditions

$$
\varphi_{\alpha 1}(\alpha ; \lambda)=\psi_{\alpha 2}(\alpha ; \lambda)=1 \quad \text { and } \quad \varphi_{\alpha 2}(\alpha ; \lambda)=\psi_{\alpha 1}(\alpha ; \lambda)=0,
$$

where $\alpha \in[0,1]$. The next lemma gives some estimates for the growth of $\varphi_{0 j}(x ; \lambda)$ and $\psi_{0 j}(x ; \lambda), j=1,2$.

Lemma 2.1. The functions $\varphi_{0 j}(x ; \lambda)$ and $\psi_{0 j}(x ; \lambda), j=0,1$, satisfy the estimates (as $\lambda \rightarrow \infty)$

$$
\begin{aligned}
& \varphi_{01}(x ; \lambda)=\left(1+O\left(\frac{1}{\Im \lambda}\right)\right) \exp (a \lambda i x), \quad \varphi_{02}(x ; \lambda)=O\left(\frac{1}{\Im \lambda}\right) \exp (a \lambda i x) \\
& \psi_{01}(x ; \lambda)=O\left(\frac{1}{\Im \lambda}\right) \exp (a \lambda i x), \quad \psi_{02}(x ; \lambda)=O\left(\frac{1}{\Im \lambda}\right) \exp (a \lambda i x) ;
\end{aligned}
$$

when $\lambda \in \mathbb{C}^{+}$, and the estimates

$$
\begin{array}{ll}
\varphi_{01}(x ; \lambda)=O\left(\frac{1}{\Im \lambda}\right) \exp (b \lambda i x), & \varphi_{02}(x ; \lambda)=O\left(\frac{1}{\Im \lambda}\right) \exp (b \lambda i x) ; \\
\psi_{01}(x ; \lambda)=O\left(\frac{1}{\Im \lambda}\right) \exp (b \lambda i x), & \psi_{02}(x ; \lambda)=\left(1+O\left(\frac{1}{\Im \lambda}\right)\right) \exp (b \lambda i x) ;
\end{array}
$$

when $\lambda \in \mathbb{C}^{-}$.

Proof. We prove the first of the estimates in (2.3). All the other estimates can be proved similarly.

According to [9] the system (1.6) admits a triangular transformation operator. This means that the solution $\varphi_{0}$ admits a representation

$$
\varphi_{0}(x ; \lambda)=\left(\begin{array}{c}
e^{i a \lambda x} \\
0
\end{array}\right)+\int_{0}^{x} K(x, t)\left(\begin{array}{c}
e^{i a \lambda t} \\
e^{i b \lambda t}
\end{array}\right) d t,
$$


where $K(x, t):=\left(\begin{array}{ll}K_{11}(x, t) & K_{12}(x, t) \\ K_{21}(x, t) & K_{22}(x, t)\end{array}\right) \in L_{\infty}(\Omega) \otimes \mathbb{C}^{2 \times 2}$. In particular,

$$
\varphi_{01}(x ; \lambda)=e^{i a \lambda x}+\int_{0}^{x} K_{11}(x, t) e^{i a \lambda t} d t+\int_{0}^{x} K_{12}(x, t) e^{i b \lambda t} d t .
$$

If $\Im \lambda>0$, then $\left|e^{i b \lambda t}\right|<1$ and $\int_{0}^{x} K_{12}(x, t) e^{i b \lambda t} d t=O(1)$, since $a<0<b$ and $K_{12}(x, t)$ is bounded, and then, in particular, $\int_{0}^{x} K_{12}(x, t) e^{i b \lambda t} d t=O\left(\frac{1}{\Im \lambda}\right) \exp (a \lambda i x)$.

Moreover, since $K_{11}(x, t)$ is bounded, one has

$$
\int_{0}^{x} K_{11}(x, t) e^{i a \lambda t} d t=O\left(\int_{0}^{x}\left|e^{i a \lambda t}\right| d t\right)=O\left(\int_{0}^{x} e^{-a(\Im \lambda) t} d t\right)=O\left(\left|\frac{1}{\Im \lambda}\right|\right) e^{i a \lambda x} .
$$

Therefore, for $\Im \lambda>0$ one gets

$$
\varphi_{01}(x ; \lambda)=e^{i a \lambda x}+O\left(\left|\frac{e^{i a \lambda x}}{\Im \lambda}\right|\right)=\left(1+O\left(\frac{1}{\Im \lambda}\right)\right) e^{i a \lambda x} .
$$

If the function $Q(x)$ is differentiable, the above estimates can be strengthened as follows.

Lemma 2.2. Let $Q(x)$ be differentiable. Then the functions $\vec{\varphi}_{j}(x ; \lambda)$ and $\vec{\psi}_{j}(x ; \lambda)$, $j \in\{0,1\}$ satisfy the estimates

$$
\begin{aligned}
& \varphi_{01}(x ; \lambda)=\exp (a \lambda i x)+\frac{1}{\lambda} O(\exp (a \lambda i x)), \quad \varphi_{02}(x ; \lambda)=\frac{1}{\lambda} O(\exp (a \lambda i x)) \\
& \psi_{01}(x ; \lambda)=\frac{1}{\lambda} O(\exp (a \lambda i x)), \quad \psi_{02}(x ; \lambda)=\frac{1}{\lambda} O(\exp (a \lambda i x)) ; \\
& \varphi_{11}(x ; \lambda)=\frac{1}{\lambda} O(\exp (b \lambda i(x-1))), \quad \varphi_{12}(x ; \lambda)=\frac{1}{\lambda} O(\exp (b \lambda i(x-1))) ; \\
& \psi_{11}(x ; \lambda)=\frac{1}{\lambda} O(\exp (b \lambda i(x-1))), \quad \psi_{12}(x ; \lambda)=\exp (b \lambda i(x-1))+\frac{1}{\lambda} O(\exp (b \lambda i(x-1))),
\end{aligned}
$$

when $\lambda \in \mathbb{C}^{+}$, and the estimates

$$
\begin{aligned}
& \varphi_{01}(x ; \lambda)=\frac{1}{\lambda} O(\exp (b \lambda i x)), \quad \varphi_{02}(x ; \lambda)=\frac{1}{\lambda} O(\exp (b \lambda i x)) ; \\
& \psi_{01}(x ; \lambda)=\frac{1}{\lambda} O(\exp (b \lambda i x)), \quad \psi_{02}(x ; \lambda)=\exp (b \lambda i x)+\frac{1}{\lambda} O(\exp (b \lambda i x)) ; \\
& \varphi_{11}(x ; \lambda)=\exp (a \lambda i(x-1))+\frac{1}{\lambda} O(\exp (a \lambda i(x-1))), \quad \varphi_{12}(x ; \lambda)=\frac{1}{\lambda} O(\exp (a \lambda i(x-1))) ; \\
& \psi_{11}(x ; \lambda)=\frac{1}{\lambda} O(\exp (a \lambda i(x-1))), \quad \psi_{12}(x ; \lambda)=\frac{1}{\lambda} O(\exp (a \lambda i(x-1))),
\end{aligned}
$$

when $\lambda \in \mathbb{C}^{-}$.

Proof. As in Lemma 2.1 we just prove the first of the estimates in (2.6). For this purpose the formula (2.5) in the proof of Lemma 2.1 will be used. In [9] it was shown that if $q_{j} \in C^{1}[0,1]$ then $K_{i j}(x, t) \in C^{1}(\Omega)$, too. Now integration by parts yields

$$
\begin{aligned}
\int_{0}^{x} K_{11}(x, t) e^{i a \lambda t} d t=\frac{1}{i a \lambda}\left(\left.K_{11}(x, t) e^{i a \lambda t}\right|_{t=0} ^{x}-\int_{0}^{x} \frac{\partial}{\partial t} K_{11}(x, t) e^{i a \lambda t} d t\right) \\
=\frac{K_{11}(x, x) e^{i a \lambda x}-K_{11}(x, 0)}{i a \lambda}-\frac{1}{i a \lambda} O\left(\left|\frac{e^{-a(\Im \lambda) x}-1}{\Im \lambda}\right|\right)=\frac{1}{\lambda} O\left(\left|e^{i a \lambda x}\right|\right),
\end{aligned}
$$


and

$$
\begin{aligned}
\int_{0}^{x} K_{12}(x, t) e^{i b \lambda t} d t=\frac{1}{i b \lambda}\left(\left.K_{12}(x, t) e^{i b \lambda t}\right|_{t=0} ^{x}-\int_{0}^{x} \frac{\partial}{\partial t} K_{12}(x, t) e^{i b \lambda t} d t\right) & =\frac{1}{i b \lambda} O(1)=\frac{1}{\lambda} O\left(\left|e^{i a \lambda x}\right|\right),
\end{aligned}
$$

since $\Im \lambda>0$ and $a<0<b$.

Therefore from the formula (2.5) one gets

$$
\varphi_{01}(x ; \lambda)=\exp (a \lambda i x)+\frac{1}{\lambda} O(\exp (a \lambda i x)) .
$$

All the other estimates in Lemma 2.2 are proved in a similar manner.

In the next lemma some estimates for the growth of the Wronski determinant are presented.

Lemma 2.3. Let $y_{1}(x ; \lambda)$ and $y_{2}(x ; \lambda)$ be two linearly independent solutions of the system (1.6). Then the Wronski determinant

$$
W(x ; \lambda)=\operatorname{det}\left(\begin{array}{ll}
y_{11}(x ; \lambda) & y_{21}(x ; \lambda) \\
y_{12}(x ; \lambda) & y_{22}(x ; \lambda)
\end{array}\right)
$$

admits the following estimate

$$
W(x ; \lambda)=(1+o(1)) \exp ((a+b) \lambda i) W(0 ; \lambda) .
$$

Proof. It was proved in [10] that in $\mathbb{C}_{ \pm}$the system (1.6) has two linearly independent solutions $\varepsilon_{1}(x, \lambda)$ and $\varepsilon_{2}(x, \lambda)$ satisfying the estimates

$$
\varepsilon_{1}(x, \lambda)=\left(\begin{array}{c}
(1+o(1)) \exp (a \lambda i x) \\
o(1) \exp (a \lambda i x)
\end{array}\right) \quad \text { and } \quad \varepsilon_{2}(x, \lambda)=\left(\begin{array}{c}
o(1) \exp (b \lambda i x) \\
(1+o(1)) \exp (b \lambda i x)
\end{array}\right)
$$

for $\lambda \in \mathbb{C}_{ \pm}$.

Since $y_{j}(x ; \lambda)$ is a linear combination of $\varepsilon_{1}(x, \lambda)$ and $\varepsilon_{2}(x, \lambda)$, one has

$$
\begin{gathered}
\left(\begin{array}{ll}
y_{11}(x ; \lambda) & y_{21}(x ; \lambda) \\
y_{12}(x ; \lambda) & y_{22}(x ; \lambda)
\end{array}\right)= \\
\left(\begin{array}{ll}
\varepsilon_{11}(x ; \lambda) & \varepsilon_{21}(x ; \lambda) \\
\varepsilon_{12}(x ; \lambda) & \varepsilon_{22}(x ; \lambda)
\end{array}\right)\left(\begin{array}{ll}
\varepsilon_{11}(0 ; \lambda) & \varepsilon_{21}(0 ; \lambda) \\
\varepsilon_{12}(0 ; \lambda) & \varepsilon_{22}(0 ; \lambda)
\end{array}\right)^{-1}\left(\begin{array}{ll}
y_{11}(0 ; \lambda) & y_{21}(0 ; \lambda) \\
y_{12}(0 ; \lambda) & y_{22}(0 ; \lambda)
\end{array}\right)
\end{gathered}
$$

It follows that

$$
\begin{array}{r}
W(x ; \lambda)=\operatorname{det}\left(\begin{array}{rr}
\varepsilon_{11}(x ; \lambda) & \varepsilon_{21}(x ; \lambda) \\
\varepsilon_{12}(x ; \lambda) & \varepsilon_{22}(x ; \lambda)
\end{array}\right) \operatorname{det}\left(\begin{array}{rr}
\varepsilon_{11}(0 ; \lambda) & \varepsilon_{21}(0 ; \lambda) \\
\varepsilon_{12}(0 ; \lambda) & \varepsilon_{22}(0 ; \lambda)
\end{array}\right)^{-1} W(0 ; \lambda) \\
=(1+o(1)) \exp ((a+b) \lambda i) W(0 ; \lambda)
\end{array}
$$

which gives the required estimate (2.9).

The function $\chi(\lambda)$ defined by

$$
\chi(\lambda):=\operatorname{det}\left(\begin{array}{ll}
Q_{11}(\lambda) & Q_{12}(\lambda) \\
Q_{21}(\lambda) & Q_{22}(\lambda)
\end{array}\right),
$$


where

$$
\begin{aligned}
& Q_{11}(\lambda)=P_{11}(\lambda)+P_{13}(\lambda) \varphi_{01}(1 ; \lambda)+P_{14}(\lambda) \varphi_{02}(1 ; \lambda), \\
& Q_{12}(\lambda)=P_{12}(\lambda)+P_{13}(\lambda) \psi_{01}(1 ; \lambda)+P_{14}(\lambda) \psi_{02}(1 ; \lambda), \\
& Q_{21}(\lambda)=P_{21}(\lambda)+P_{23}(\lambda) \varphi_{01}(1 ; \lambda)+P_{24}(\lambda) \varphi_{02}(1 ; \lambda), \\
& Q_{22}(\lambda)=P_{22}(\lambda)+P_{23}(\lambda) \psi_{01}(1 ; \lambda)+P_{24}(\lambda) \psi_{02}(1 ; \lambda),
\end{aligned}
$$

is said to be the characteristic function of the problem (1.6), (1.7). This definition is motivated by the next result.

Proposition 2.4. The number $\lambda_{0} \in \mathbb{C}$ is an eigenvalue of the operator associated with the problem (1.6), (1.7) if and only if $\chi\left(\lambda_{0}\right)=0$. Moreover, the functions $\overrightarrow{\omega_{1}}(x ; \lambda)$ and $\overrightarrow{\omega_{2}}(x ; \lambda)$ given by

$$
\overrightarrow{\omega_{1}}(x ; \lambda)=Q_{12}(\lambda) \varphi_{0}(x ; \lambda)-Q_{11}(\lambda) \psi_{0}(x ; \lambda)
$$

and

$$
\overrightarrow{\omega_{2}}(x ; \lambda)=Q_{22}(\lambda) \varphi_{0}(x ; \lambda)-Q_{21}(\lambda) \psi_{0}(x ; \lambda)
$$

are the eigenfunctions corresponding to the eigenvalue $\lambda_{0}$, or, one has $\overrightarrow{\omega_{j}}(x ; \lambda) \equiv 0$. Moreover, all the eigenfunctions and associate functions corresponding to the eigenvalue $\lambda_{0}$ are the nonzero functions of the form

$$
\left.\frac{1}{k !} \frac{\partial^{k}}{\partial \lambda^{k}} \omega_{j}(x ; \lambda)\right|_{\lambda=\lambda_{0}}, \quad \text { where } 0 \leq k<p_{j}, \quad j=1,2 .
$$

Proof. It follows from (2.2) with $\alpha=0$ that for all $\lambda \in \mathbb{C}$ the function

$$
\begin{aligned}
\overrightarrow{\omega_{1}}(x ; \lambda)= & \left(P_{12}(\lambda)+P_{13}(\lambda) \psi_{01}(1 ; \lambda)+P_{14}(\lambda) \psi_{02}(1 ; \lambda)\right) \varphi_{0}(x ; \lambda) \\
& -\left(P_{11}(\lambda)+P_{13}(\lambda) \varphi_{01}(1 ; \lambda)+P_{14}(\lambda) \varphi_{02}(1 ; \lambda)\right) \psi_{0}(x ; \lambda)
\end{aligned}
$$

is a solution of the first equation in (1.7). Moreover, since

$$
P_{21}(\lambda) \omega_{11}(0, \lambda)+P_{22}(\lambda) \omega_{12}(0, \lambda)+P_{23}(\lambda) \omega_{11}(1, \lambda)+P_{24}(\lambda) \omega_{12}(1, \lambda)=-\chi(\lambda),
$$

$\overrightarrow{\omega_{1}}\left(x ; \lambda_{0}\right)$ is a solution of the second equation in (1.7), if $\lambda_{0}$ is a root of $\chi(\lambda)$. Similarly, for all $\lambda \in \mathbb{C}$ the function

$$
\begin{aligned}
\overrightarrow{\omega_{2}}(x ; \lambda)= & \left(P_{22}(\lambda)+P_{23}(\lambda) \psi_{01}(1 ; \lambda)+P_{24}(\lambda) \psi_{02}(1 ; \lambda)\right) \varphi_{0}(x ; \lambda) \\
& -\left(P_{21}(\lambda)+P_{23}(\lambda) \varphi_{01}(1 ; \lambda)+P_{24}(\lambda) \varphi_{02}(1 ; \lambda)\right) \psi_{0}(x ; \lambda) .
\end{aligned}
$$

is a solution of the second equation in (1.7) and since

$$
P_{11}(\lambda) \omega_{21}(0, \lambda)+P_{12}(\lambda) \omega_{22}(0, \lambda)+P_{13}(\lambda) \omega_{21}(1, \lambda)+P_{14}(\lambda) \omega_{22}(1, \lambda)=\chi(\lambda),
$$

$\overrightarrow{\omega_{j}}\left(x ; \lambda_{0}\right)$ is a solution of the first equation in (1.7) too, if $\lambda_{0}$ is a root of $\chi(\lambda)$.

If, in addition, $\lambda_{0}$ is a root of $\chi(\lambda)$ of the order $p_{0}=p_{0}^{(1)}+p_{0}^{(2)}$, then the operator determined by (1.6), (1.7) has precisely $p$ eigenfunctions and associate functions corresponding to $\lambda_{0}$. In fact, it follows from (2.19), (2.21) that all nonzero functions

$$
\left.\frac{1}{k !} \frac{\partial^{k}}{\partial \lambda^{k}} \omega_{j}(x ; \lambda)\right|_{\lambda=\lambda_{0}}, \quad \text { where } \min \left(p_{0}^{(1)}, p_{0}^{(2)}\right) \leq k<p_{0}, \quad j=1,2,
$$

are eigenfunctions and associate functions corresponding to the eigenvalue $\lambda_{0}$.

The completeness result can now be stated as follows. 
Theorem 2.5. Let $P_{i j}(i=1,2 ; j=1,2,3,4)$ be polynomials, let the rank of the polynomial matrix

$$
P(\lambda)=\left(\begin{array}{llll}
P_{11}(\lambda) & P_{12}(\lambda) & P_{13}(\lambda) & P_{14}(\lambda) \\
P_{21}(\lambda) & P_{22}(\lambda) & P_{23}(\lambda) & P_{24}(\lambda)
\end{array}\right)
$$

be equal to 2 for all $\lambda \in \mathbb{C}$, and let

$$
\operatorname{deg} J_{14}=\operatorname{deg} J_{32} \geq \max \left\{\operatorname{deg} J_{13}, \operatorname{deg} J_{42}, M\right\},
$$

where $M=\max \left\{\operatorname{deg} P_{i j}: i \in\{1,2\} ; j \in\{1,2,3,4\}\right\}$ and

$$
J_{i j}=\operatorname{det}\left(\begin{array}{ll}
P_{1 i} & P_{1 j} \\
P_{2 i} & P_{2 j}
\end{array}\right), \quad i, j \in\{1,2,3,4\} .
$$

Then the SEAF of the problem (1.6), (1.7) is complete in $L^{2}[0,1] \oplus L^{2}[0,1]$.

Moreover, let the set $\Phi$, which consists of $N:=\operatorname{deg} J_{14}-M$ eigenfunctions and associate functions, satisfy the following condition:

If $\Phi$ contains either an eigenfunction or an associate function corresponding to an eigenvalue $\lambda_{k}$, then it also contains all the associate functions of higher order corresponding to the same eigenvalue.

Then the SEAF of the problem (1.6), (1.7) without the set $\Phi$ is also complete in the space $L^{2}[0,1] \oplus L^{2}[0,1]$.

Proof. Suppose that the SEAF of the problem (1.6), (1.7) without the set $\Phi$ is not complete in the space $L^{2}[0,1] \oplus L^{2}[0,1]$. Then there exists a nonzero vector function $\vec{f}(x)=$ $\left(f_{1}(x), f_{2}(x)\right)^{\top}$, which is orthogonal to the SEAF of the problem (1.6), (1.7) (possibly, excluding functions from the set $\Phi)$. Define

$$
\widetilde{F}_{j}(\lambda):=\left\langle\vec{\omega}_{j}(x ; \lambda), \vec{f}(x)\right\rangle=\int_{0}^{1}\left(\omega_{j 1}(x ; \lambda) \overline{f_{1}(x)}+\omega_{j 2}(x ; \lambda) \overline{f_{2}(x)}\right) d x .
$$

Clearly, $\widetilde{F_{j}}(\lambda)$ is an entire function. If $\lambda_{s}$ is an eigenvalue of multiplicity $p_{s}=p_{s}^{(1)}+p_{s}^{(2)}$ and the set $\Phi$ contains neither an eigenfunction nor an associate function corresponding to $\lambda_{s}$, then it follows from Proposition 2.4 that $\lambda_{s}$ is a root of $\widetilde{F_{j}}(\lambda)$ of order $p_{s}^{(j)}, j=1,2$.

If $\Phi$ contains $k$ eigenfunctions or associate functions corresponding to the eigenvalue $\lambda_{s}$, then $\lambda_{s}$ is a root of $\widetilde{F_{j}}(\lambda)$ of order greater than or equal to $p_{s}^{(j)}-k, j=1,2$.

Let $\Phi \neq \emptyset$ and denote by $\Lambda$ the set of all eigenvalues of the problem (1.6), (1.7), such that the corresponding eigenfunctions (or associate functions) belong to the set $\Phi$. For each $\lambda_{s} \in \Lambda$ denote by $q_{s}$ the number of eigenfunctions and associate functions in $\Phi$ corresponding to $\lambda_{s}$. Define

$$
\Pi(\lambda)=\prod_{\lambda_{s} \in \Lambda}\left(\lambda-\lambda_{s}\right)^{q_{s}}
$$

Let $\lambda_{k}$ be an eigenvalue of the problem (1.6), (1.7) of multiplicity $p_{k}$. Then $\lambda_{k}$ is a zero of the product $\Pi(\lambda) \widetilde{F}_{j}(\lambda)$ at least of order $p_{k}$. Consequently, the functions

$$
F_{j}(\lambda)=\frac{\Pi(\lambda) \widetilde{F_{j}}(\lambda)}{\chi(\lambda)}
$$

are entire. Next an estimate for these functions will be derived. 
One can rewrite $\chi(\lambda)$ as follows

$$
\begin{aligned}
\chi(\lambda)= & J_{12}+J_{13} \psi_{01}(1 ; \lambda)+J_{14} \psi_{02}(1 ; \lambda)+J_{32} \varphi_{01}(1 ; \lambda) \\
& +J_{42} \varphi_{02}(1 ; \lambda)+J_{34} \operatorname{det}\left(\begin{array}{cc}
\varphi_{01}(1 ; \lambda) & \psi_{01}(1 ; \lambda) \\
\varphi_{02}(1 ; \lambda) & \psi_{02}(1 ; \lambda)
\end{array}\right) \\
= & J_{12}+J_{13} \psi_{01}(1 ; \lambda)+J_{14} \psi_{02}(1 ; \lambda)+J_{32} \varphi_{01}(1 ; \lambda) \\
& +J_{42} \varphi_{02}(1 ; \lambda)+J_{34}\left(1+O\left(\frac{1}{\Im \lambda}\right)\right) \exp ((a+b) \lambda i) .
\end{aligned}
$$

Then one obtains from $(2.3),(2.4)$, and the assumption (2.24) the following estimates for $\chi(\lambda)$ :

$$
\begin{array}{ll}
\chi(\lambda)=\left(1+O\left(\frac{1}{\Im \lambda}\right)\right) J_{32} \exp (a \lambda i), & \lambda \in \mathbb{C}^{+} ; \\
\chi(\lambda)=\left(1+O\left(\frac{1}{\Im \lambda}\right)\right) J_{14} \exp (b \lambda i), & \lambda \in \mathbb{C}^{-} .
\end{array}
$$

Moreover, the definition of $\vec{\omega}_{j}(x ; \lambda)$ (cf. (2.18) and (2.20)) implies that

$$
\begin{aligned}
\vec{\omega}_{j}(x ; \lambda)=- & P_{j 1}(\lambda) \psi_{0}(x ; \lambda)+P_{j 2}(\lambda) \varphi_{0}(x ; \lambda) \\
& +\left(1+O\left(\frac{1}{\Im \lambda}\right)\right) \exp ((a+b) \lambda i)\left(-P_{j 3}(\lambda) \psi_{1}(x ; \lambda)+P_{j 4}(\lambda) \varphi_{1}(x ; \lambda)\right) .
\end{aligned}
$$

If $\lambda \in \mathbb{C}^{+}$, then (2.32) and the estimate (2.3) imply

$$
\begin{aligned}
\omega_{j k}(x ; \lambda)=( & \left.O\left(P_{j 1}(\lambda)\right)+O\left(P_{j 2}(\lambda)\right)\right) \exp (a \lambda i x) \\
& +\left(O\left(P_{j 3}(\lambda)\right)+O\left(P_{j 4}(\lambda)\right)\right) \exp (a \lambda i) \exp (b \lambda i x) .
\end{aligned}
$$

By using the Cauchy-Schwartz inequality one gets

$$
\int_{0}^{1}\left|f_{k}(x) \exp (a \lambda i x)\right| d x=O\left(\frac{\exp (a \lambda i)}{\sqrt{|\Im \lambda|}}\right), \quad \int_{0}^{1}\left|f_{k}(x) \exp (b \lambda i x)\right| d x=O\left(\frac{1}{\sqrt{|\Im \lambda|}}\right),
$$

and consequently there exists a constant $c_{1}>0$, such that, for $\Im \lambda>c_{1}$ and $a<0<b$,

$$
\tilde{F}_{j}(\lambda)=O\left(\max \left|P_{j k}(\lambda)\right| \frac{\exp (a \lambda i)}{\sqrt{|\Im \lambda|}}\right)=O\left(\frac{\lambda^{M}}{\sqrt{|\Im \lambda|}}\right) e^{a \lambda i} .
$$

Similarly, there exists a constant $c_{2}<0$, such that, for $\Im \lambda<c_{2}$,

$$
\widetilde{F}_{j}(\lambda)=O\left(\frac{\lambda^{M}}{\sqrt{|\Im \lambda|}}\right) e^{b \lambda i}
$$

From (2.30), (2.31), (2.34), (2.35), and the assumption (2.24) one obtains finally the estimate

$$
F_{j}(\lambda)=O\left(\frac{1}{\sqrt{|\Im \lambda|}}\right), \quad|\Im \lambda|>c .
$$

By applying Phragmen-Lindelöf theorem for a strip one concludes that $F_{j}(\lambda) \equiv 0$. Consequently, $\widetilde{F}_{j}(\lambda) \equiv 0$, i.e. $\vec{f}(x)$ is orthogonal to $\vec{\omega}_{1}(x ; \lambda)$ and $\vec{\omega}_{2}(x ; \lambda)$ for all $\lambda$. However, the functions $\vec{\omega}_{1}(x ; \lambda)$ and $\vec{\omega}_{2}(x ; \lambda)$ for all $\lambda$ form a fundamental system of solutions of the equation (1.6) if $\lambda$ is not an eigenvalue. Since the set of eigenvalues coincides with the set of all roots of $\chi(\lambda)$, this set is discrete. This implies that $\vec{f}(x)$ is orthogonal to all solutions of the equation (1.6), so that $\vec{f}(x) \equiv 0$.

Therefore, there is no nontrivial function $\vec{f}(x)$ orthogonal to the SEAF of the problem (1.6)-(1.7) (maybe without the set $\Phi)$. 
Theorem 2.6. Let $P_{i j}(i=1,2 ; j=0,1, \ldots, 9)$ be polynomials, let the rank of the matrix

$$
\left(\begin{array}{llll}
P_{10}(\lambda) & P_{11}(\lambda) & \ldots & P_{19}(\lambda) \\
P_{20}(\lambda) & P_{21}(\lambda) & \ldots & P_{29}(\lambda)
\end{array}\right)
$$

be equal to 2 for all $\lambda \in \mathbb{C}$, and let

$$
\operatorname{deg} J_{03}=\operatorname{deg} J_{12}=M,
$$

where

$$
J_{i j}=\operatorname{det}\left(\begin{array}{ll}
P_{1 i} & P_{1 j} \\
P_{2 i} & P_{2 j}
\end{array}\right), \quad i, j=0,1, \ldots, 9,
$$

and $M=\max \left\{\operatorname{deg} P_{i j}: i \in\{1,2\} ; j \in\{0,1, \ldots, 9\}\right\}$. Then the SEAF of the problem (1.6), (1.8) is complete in $L^{2}[0,1] \oplus L^{2}[0,1]$.

Moreover, let the set $\Phi$, which consists of $M$ eigenfunctions and associate functions, satisfy the following condition:

If $\Phi$ contains either an eigenfunction or an associate function corresponding to an eigenvalue $\lambda_{k}$, then it also contains all the associate functions of higher order corresponding to the same eigenvalue.

Then the SEAF of the problem (1.6), (1.8) without the set $\Phi$ is also complete in the space $L^{2}[0,1] \oplus L^{2}[0,1]$.

Proof. The proof of this theorem is similar to the proof of Theorem 2.5.

As in Theorem 2.5 one considers the characteristic function and the functions $\vec{\omega}_{1}(x ; \lambda)$ and $\vec{\omega}_{2}(x ; \lambda)$, but in this case these functions will be defined by other formulas.

Let $\vec{\omega}(x ; \lambda)$ be an arbitrary solution of the system $(1.6)$. Then $\vec{\omega}(x ; \lambda)$ may be written in the form

$$
\vec{\omega}(x ; \lambda)=A \overrightarrow{\varphi_{0}}(x ; \lambda)+B \overrightarrow{\psi_{0}}(x ; \lambda) .
$$

It follows that $\vec{\omega}(x ; \lambda)$ is a solution of the problem (1.6), (1.8) if and only if

$$
\left\{\begin{array}{r}
P_{10}(\lambda) A^{2}+P_{11}(\lambda) B^{2}+P_{12}(\lambda)\left(A \varphi_{01}\left(\frac{1}{2} ; \lambda\right)+B \psi_{01}\left(\frac{1}{2} ; \lambda\right)\right)^{2}+ \\
P_{13}(\lambda)\left(A \varphi_{02}\left(\frac{1}{2} ; \lambda\right)+B \psi_{02}\left(\frac{1}{2} ; \lambda\right)\right)^{2}+P_{14}(\lambda) A B+ \\
P_{15}(\lambda) A\left(A \varphi_{01}\left(\frac{1}{2} ; \lambda\right)+B \psi_{01}\left(\frac{1}{2} ; \lambda\right)\right)+P_{16}(\lambda) A\left(A \varphi_{02}\left(\frac{1}{2} ; \lambda\right)+B \psi_{02}\left(\frac{1}{2} ; \lambda\right)\right)+ \\
P_{17}(\lambda) B\left(A \varphi_{01}\left(\frac{1}{2} ; \lambda\right)+B \psi_{01}\left(\frac{1}{2} ; \lambda\right)\right)+P_{18}(\lambda) B\left(A \varphi_{02}\left(\frac{1}{2} ; \lambda\right)+B \psi_{02}\left(\frac{1}{2} ; \lambda\right)\right)+ \\
P_{19}(\lambda)\left(A \varphi_{01}\left(\frac{1}{2} ; \lambda\right)+B \psi_{01}\left(\frac{1}{2} ; \lambda\right)\right)\left(A \varphi_{02}\left(\frac{1}{2} ; \lambda\right)+B \psi_{02}\left(\frac{1}{2} ; \lambda\right)\right)=0, \\
P_{20}(\lambda) A^{2}+P_{21}(\lambda) B^{2}+P_{22}(\lambda)\left(A \varphi_{01}\left(\frac{1}{2} ; \lambda\right)+B \psi_{01}\left(\frac{1}{2} ; \lambda\right)\right)^{2}+ \\
P_{23}(\lambda)\left(A \varphi_{02}\left(\frac{1}{2} ; \lambda\right)+B \psi_{02}\left(\frac{1}{2} ; \lambda\right)\right)^{2}+P_{24}(\lambda) A B+ \\
P_{25}(\lambda) A\left(A \varphi_{01}\left(\frac{1}{2} ; \lambda\right)+B \psi_{01}\left(\frac{1}{2} ; \lambda\right)\right)+P_{26}(\lambda) A\left(A \varphi_{02}\left(\frac{1}{2} ; \lambda\right)+B \psi_{02}\left(\frac{1}{2} ; \lambda\right)\right)+ \\
P_{27}(\lambda) B\left(A \varphi_{01}\left(\frac{1}{2} ; \lambda\right)+B \psi_{01}\left(\frac{1}{2} ; \lambda\right)\right)+P_{28}(\lambda) B\left(A \varphi_{02}\left(\frac{1}{2} ; \lambda\right)+B \psi_{02}\left(\frac{1}{2} ; \lambda\right)\right)+ \\
P_{29}(\lambda)\left(A \varphi_{01}\left(\frac{1}{2} ; \lambda\right)+B \psi_{01}\left(\frac{1}{2} ; \lambda\right)\right)\left(A \varphi_{02}\left(\frac{1}{2} ; \lambda\right)+B \psi_{02}\left(\frac{1}{2} ; \lambda\right)\right)=0 .
\end{array}\right.
$$

The system (2.40) may be rewritten in the form

$$
\left\{\begin{array}{l}
Q_{11}(\lambda) A^{2}+Q_{12}(\lambda) A B+Q_{13}(\lambda) B^{2}=0 \\
Q_{21}(\lambda) A^{2}+Q_{22}(\lambda) A B+Q_{23}(\lambda) B^{2}=0
\end{array}\right.
$$


where

$$
\begin{aligned}
Q_{11}= & P_{10}(\lambda)+P_{12}(\lambda) \varphi_{01}^{2}\left(\frac{1}{2} ; \lambda\right)+P_{13}(\lambda) \varphi_{02}^{2}\left(\frac{1}{2} ; \lambda\right)+ \\
& +P_{15}(\lambda) \varphi_{01}\left(\frac{1}{2} ; \lambda\right)+P_{16}(\lambda) \varphi_{02}\left(\frac{1}{2} ; \lambda\right)+P_{19}(\lambda) \varphi_{01}\left(\frac{1}{2} ; \lambda\right) \varphi_{02}\left(\frac{1}{2} ; \lambda\right), \\
Q_{12}= & 2 P_{12}(\lambda) \varphi_{01}\left(\frac{1}{2} ; \lambda\right) \psi_{01}\left(\frac{1}{2} ; \lambda\right)+2 P_{13}(\lambda) \varphi_{02}\left(\frac{1}{2} ; \lambda\right) \psi_{02}\left(\frac{1}{2} ; \lambda\right)+P_{14}(\lambda)+ \\
& +P_{15}(\lambda) \psi_{01}\left(\frac{1}{2} ; \lambda\right)+P_{16}(\lambda) \psi_{02}\left(\frac{1}{2} ; \lambda\right)+P_{17}(\lambda) \varphi_{01}\left(\frac{1}{2} ; \lambda\right)+ \\
& +P_{18}(\lambda) \varphi_{02}\left(\frac{1}{2} ; \lambda\right)+P_{19}(\lambda)\left(\varphi_{01}\left(\frac{1}{2} ; \lambda\right) \psi_{02}\left(\frac{1}{2} ; \lambda\right)+\psi_{01}\left(\frac{1}{2} ; \lambda\right) \varphi_{02}\left(\frac{1}{2} ; \lambda\right)\right), \\
& \left.Q_{13}(\lambda)+P_{12}(\lambda) \psi_{01}^{2}\left(\frac{1}{2} ; \lambda\right)\right)+P_{13}(\lambda) \psi_{02}^{2}\left(\frac{1}{2} ; \lambda\right)+ \\
& \left.+P_{17}(\lambda) \psi_{01}\left(\frac{1}{2} ; \lambda\right)\right)+P_{18}(\lambda) \psi_{02}\left(\frac{1}{2} ; \lambda\right)+P_{19}(\lambda) \psi_{01}\left(\frac{1}{2} ; \lambda\right) \psi_{02}\left(\frac{1}{2} ; \lambda\right) ; \\
& \\
Q_{21}= & P_{20}(\lambda)+P_{22}(\lambda) \varphi_{01}^{2}\left(\frac{1}{2} ; \lambda\right)+P_{23}(\lambda) \varphi_{02}^{2}\left(\frac{1}{2} ; \lambda\right)+ \\
& +P_{25}(\lambda) \varphi_{01}\left(\frac{1}{2} ; \lambda\right)+P_{26}(\lambda) \varphi_{02}\left(\frac{1}{2} ; \lambda\right)+P_{29}(\lambda) \varphi_{01}\left(\frac{1}{2} ; \lambda\right) \varphi_{02}\left(\frac{1}{2} ; \lambda\right), \\
& \\
Q_{22}= & P_{22}(\lambda) \varphi_{01}\left(\frac{1}{2} ; \lambda\right) \psi_{01}\left(\frac{1}{2} ; \lambda\right)+2 P_{23}(\lambda) \varphi_{02}\left(\frac{1}{2} ; \lambda\right) \psi_{02}\left(\frac{1}{2} ; \lambda\right)+P_{24}(\lambda)+ \\
& +P_{25}(\lambda) \psi_{01}\left(\frac{1}{2} ; \lambda\right)+P_{26}(\lambda) \psi_{02}\left(\frac{1}{2} ; \lambda\right)+P_{27}(\lambda) \varphi_{01}\left(\frac{1}{2} ; \lambda\right)+ \\
& +P_{28}(\lambda) \varphi_{02}\left(\frac{1}{2} ; \lambda\right)+P_{29}(\lambda)\left(\varphi_{01}\left(\frac{1}{2} ; \lambda\right) \psi_{02}\left(\frac{1}{2} ; \lambda\right)+\psi_{01}\left(\frac{1}{2} ; \lambda\right) \varphi_{02}\left(\frac{1}{2} ; \lambda\right)\right), \\
Q_{23}= & \left.P_{21}(\lambda)+P_{22}(\lambda) \psi_{01}^{2}\left(\frac{1}{2} ; \lambda\right)\right)+P_{23}(\lambda) \psi_{02}^{2}\left(\frac{1}{2} ; \lambda\right)+ \\
& \left.+P_{27}(\lambda) \psi_{01}\left(\frac{1}{2} ; \lambda\right)\right)+P_{28}(\lambda) \psi_{02}\left(\frac{1}{2} ; \lambda\right)+P_{29}(\lambda) \psi_{01}\left(\frac{1}{2} ; \lambda\right) \psi_{02}\left(\frac{1}{2} ; \lambda\right)
\end{aligned}
$$

It is well known (see, for example, [23, Chap. $5, \S 34]$ ), that a system of two quadratic equations has a nonzero solution if and only if, the resultant is equal to 0 . Therefore, $\lambda_{0}$ is an eigenvalue of the problem (1.6), (1.8) if and only if $\chi(\lambda)=0$, where

$$
\chi(\lambda)=\operatorname{det}\left(\begin{array}{cccc}
Q_{11} & Q_{12} & Q_{13} & 0 \\
0 & Q_{11} & Q_{12} & Q_{13} \\
Q_{21} & Q_{22} & Q_{23} & 0 \\
0 & Q_{21} & Q_{22} & Q_{23}
\end{array}\right)=D_{13}^{2}-D_{12} D_{23}
$$

with

$$
D_{i j}=\operatorname{det}\left(\begin{array}{ll}
Q_{1 i} & Q_{1 j} \\
Q_{2 i} & Q_{2 j}
\end{array}\right) .
$$

Moreover, the multiplicity of $\lambda_{0}$ as a zero of the function $\chi(\lambda)$ is equal to the number of eigenfunctions and associate functions corresponding to the eigenvalue $\lambda_{0}$.

Introduce the Wronski determinant

$$
W(x ; \lambda)=\operatorname{det}\left(\begin{array}{ll}
\varphi_{01}(x ; \lambda) & \psi_{01}(x ; \lambda) \\
\varphi_{02}(x ; \lambda) & \psi_{02}(x ; \lambda)
\end{array}\right) .
$$

Then $\chi(\lambda)$ may be transformed to a polynomial of degree 4 with the arguments $\varphi_{01}\left(\frac{1}{2} ; \lambda\right)$, $\psi_{01}\left(\frac{1}{2} ; \lambda\right), \varphi_{02}\left(\frac{1}{2} ; \lambda\right), \psi_{02}\left(\frac{1}{2} ; \lambda\right)$, and $W\left(\frac{1}{2}, \lambda\right)$, having all the coefficients of form $J_{i j} J_{k l}$. In particular, the coefficient of $\varphi_{0,1}^{4}\left(\frac{1}{2} ; \lambda\right)$ is equal to $J_{12}^{2}$, and the coefficient of $\psi_{0,2}^{4}\left(\frac{1}{2} ; \lambda\right)$ is 
equal to $J_{03}^{2}$. Therefore, from the condition (2.38), the estimates (2.3), (2.4), and the following estimate (cf. Lemma 2.3)

$$
W(x ; \lambda)=\left(1+O\left(\frac{1}{\lambda}\right)\right) \exp ((a+b) \lambda i),
$$

one can derive the following estimates for the characteristic function $\chi(\lambda)$ :

$$
\chi(\lambda)=\left(1+O\left(\frac{1}{\Im \lambda}\right)\right) J_{12}^{2} \exp (2 a \lambda i) \quad \text { for } \lambda \in \mathbb{C}^{+}
$$

and

$$
\chi(\lambda)=\left(1+O\left(\frac{1}{\Im \lambda}\right)\right) J_{03}^{2} \exp (2 b \lambda i) \quad \text { for } \lambda \in \mathbb{C}^{-} .
$$

Now, introduce the functions

$$
\vec{\omega}_{1}(x ; \lambda):=D_{13} \varphi_{0}(x ; \lambda)-D_{12} \psi_{0}(x ; \lambda) \quad \text { and } \quad \vec{\omega}_{2}(x ; \lambda):=D_{23} \varphi_{0}(x ; \lambda)-D_{13} \psi_{0}(x ; \lambda) \text {. }
$$

The function $\vec{\omega}_{1}(x ; \lambda)$ satisfies the boundary conditions (1.8) if and only if

$$
\left\{\begin{array}{l}
\Gamma_{1}(\lambda):=Q_{11}(\lambda) D_{13}^{2}(\lambda)-Q_{12}(\lambda) D_{13}(\lambda) D_{12}(\lambda)+Q_{13}(\lambda) D_{12}^{2}(\lambda)=0 \\
\Gamma_{2}(\lambda):=Q_{21}(\lambda) D_{13}^{2}(\lambda)-Q_{22}(\lambda) D_{13}(\lambda) D_{12}(\lambda)+Q_{23}(\lambda) D_{12}^{2}(\lambda)=0
\end{array} .\right.
$$

Observe that

$$
\Gamma_{1}(\lambda)=Q_{11}(\lambda) \chi(\lambda) \text { and } \Gamma_{2}(\lambda)=Q_{21}(\lambda) \chi(\lambda)
$$

Hence, if $\lambda_{0}$ is an eigenvalue of multiplicity $p$, then

$$
\left.\frac{\partial^{k}}{\partial \lambda^{k}} \Gamma_{1}(x ; \lambda)\right|_{\lambda=\lambda_{0}}=0 \quad \text { and }\left.\frac{\partial^{k}}{\partial \lambda^{k}} \Gamma_{2}(x ; \lambda)\right|_{\lambda=\lambda_{0}}=0 \quad \text { for all } k<p .
$$

Therefore, in this case, all nonzero functions

$$
\left.\frac{\partial^{k}}{\partial \lambda^{k}} \vec{\omega}_{1}(x ; \lambda)\right|_{\lambda=\lambda_{0}}=0, \quad \text { with } k<p
$$

are eigenfunctions and associate functions, corresponding to the eigenvalue $\lambda_{0}$. Similarly all nonzero functions given by

$$
\left.\frac{\partial^{k}}{\partial \lambda^{k}} \vec{\omega}_{2}(x ; \lambda)\right|_{\lambda=\lambda_{0}}=0, \quad \text { with } k<p
$$

are eigenfunctions and associate functions, corresponding to the eigenvalue $\lambda_{0}$, too.

Suppose that the SEAF of the problem (1.6), (1.8) without the set $\Phi$ is not complete in the space $L^{2}[0,1] \oplus L^{2}[0,1]$. Then there exists a nonzero vector-function

$$
\vec{f}(x)=\left(\begin{array}{l}
f_{1}(x) \\
f_{2}(x)
\end{array}\right)
$$

which is orthogonal to the SEAF of problem (1.6)-(1.8) (possibly excluding the functions from the set $\Phi)$.

Just as in the proof of Theorem 2.5 introduce the functions $\tilde{F}_{j}(\lambda)$ and $\Pi(\lambda)$ by the formulae (2.26) and (2.27). Then, as before, the functions

$$
F_{j}(\lambda)=\frac{\Pi(\lambda) \tilde{F}_{j}(\lambda)}{\chi(\lambda)}
$$

are entire. Let

$$
\vec{g}(x)=\left(c_{1} \psi_{01}\left(\frac{1}{2} ; \lambda\right)+c_{2} \psi_{02}\left(\frac{1}{2} ; \lambda\right)\right) \overrightarrow{\varphi_{0}}(x ; \lambda)-\left(c_{1} \varphi_{01}\left(\frac{1}{2} ; \lambda\right)+c_{2} \varphi_{02}\left(\frac{1}{2} ; \lambda\right)\right) \overrightarrow{\psi_{0}}(x ; \lambda),
$$


where $c_{1}$ and $c_{2}$ are arbitrary complex coefficients. From (2.45) one gets the estimate

$$
\vec{g}\left(\frac{1}{2}\right)=\left(\begin{array}{c}
\left(-c_{1}+O\left(\frac{1}{\lambda}\right)\right) \exp ((a+b) \lambda i) \\
\left(c_{2}+O\left(\frac{1}{\lambda}\right)\right) \exp ((a+b) \lambda i)
\end{array}\right)
$$

and this implies that the function $\vec{g}(x)$ satisfies the estimate

$$
\vec{g}(x)=\left(1+O\left(\frac{1}{\Im \lambda}\right)\right) \exp \left(\frac{1}{2}(a+b) \lambda i\right)\left(-c_{1} \overrightarrow{\psi_{\frac{1}{2}}}(x ; \lambda)+c_{2} \overrightarrow{\varphi_{\frac{1}{2}}}(x ; \lambda)\right)
$$

where the functions $\overrightarrow{\varphi_{\frac{1}{2}}}(x ; \lambda)$ and $\overrightarrow{\psi_{\frac{1}{2}}}(x ; \lambda)$ are solutions of the Cauchy problem for the system (1.6) with the initial conditions

$$
\varphi_{\frac{1}{2} 1}\left(\frac{1}{2} ; \lambda\right)=\psi_{\frac{1}{2} 2}\left(\frac{1}{2} ; \lambda\right)=1 \quad \text { and } \quad \varphi_{\frac{1}{2} 2}\left(\frac{1}{2} ; \lambda\right)=\psi_{\frac{1}{2} 1}\left(\frac{1}{2} ; \lambda\right)=0 .
$$

As in Lemma 2.1 one can derive for these functions the following estimates:

if $\lambda \in \mathbb{C}^{+}$and $x>\frac{1}{2}$ then

$$
\begin{aligned}
& \varphi_{\frac{1}{2} 1}(x ; \lambda)=\left(1+O\left(\frac{1}{\Im \lambda}\right)\right) \exp \left(a \lambda i\left(x-\frac{1}{2}\right)\right), \\
& \varphi_{\frac{1}{2} 2}(x ; \lambda)=O\left(\frac{1}{\Im \lambda}\right) \exp \left(a \lambda i\left(x-\frac{1}{2}\right)\right) \\
& \psi_{\frac{1}{2} 1}(x ; \lambda)=O\left(\frac{1}{\Im \lambda}\right) \exp \left(a \lambda i\left(x-\frac{1}{2}\right)\right) \\
& \psi_{\frac{1}{2} 2}(x ; \lambda)=O\left(\frac{1}{\Im \lambda}\right) \exp \left(a \lambda i\left(x-\frac{1}{2}\right)\right)
\end{aligned}
$$

if $\lambda \in \mathbb{C}^{+}$and $x<\frac{1}{2}$ then

$$
\begin{aligned}
& \varphi_{\frac{1}{2} 1}(x ; \lambda)=O\left(\frac{1}{\Im \lambda}\right) \exp \left(b \lambda i\left(x-\frac{1}{2}\right)\right), \\
& \varphi_{\frac{1}{2} 2}(x ; \lambda)=O\left(\frac{1}{\Im \lambda}\right) \exp \left(b \lambda i\left(x-\frac{1}{2}\right)\right) ; \\
& \psi_{\frac{1}{2} 1}(x ; \lambda)=O\left(\frac{1}{\Im \lambda}\right) \exp \left(b \lambda i\left(x-\frac{1}{2}\right)\right), \\
& \psi_{\frac{1}{2} 2}(x ; \lambda)=\left(1+O\left(\frac{1}{\Im \lambda}\right)\right) \exp \left(b \lambda i\left(x-\frac{1}{2}\right)\right) ;
\end{aligned}
$$

if $\lambda \in \mathbb{C}^{-}$and $x>\frac{1}{2}$ then

$$
\begin{aligned}
& \varphi_{\frac{1}{2} 1}(x ; \lambda)=O\left(\frac{1}{\Im \lambda}\right) \exp \left(b \lambda i\left(x-\frac{1}{2}\right)\right), \\
& \varphi_{\frac{1}{2} 2}(x ; \lambda)=O\left(\frac{1}{\Im \lambda}\right) \exp \left(b \lambda i\left(x-\frac{1}{2}\right)\right) ; \\
& \psi_{\frac{1}{2} 1}(x ; \lambda)=O\left(\frac{1}{\Im \lambda}\right) \exp \left(b \lambda i\left(x-\frac{1}{2}\right)\right), \\
& \psi_{\frac{1}{2} 2}(x ; \lambda)=\left(1+O\left(\frac{1}{\Im \lambda}\right)\right) \exp \left(b \lambda i\left(x-\frac{1}{2}\right)\right) ;
\end{aligned}
$$


if $\lambda \in \mathbb{C}^{-}$and $x<\frac{1}{2}$ then

$$
\begin{aligned}
& \varphi_{\frac{1}{2} 1}(x ; \lambda)=\left(1+O\left(\frac{1}{\Im \lambda}\right)\right) \exp \left(b \lambda i\left(x-\frac{1}{2}\right)\right), \\
& \varphi_{\frac{1}{2} 2}(x ; \lambda)=O\left(\frac{1}{\Im \lambda}\right) \exp \left(b \lambda i\left(x-\frac{1}{2}\right)\right) ; \\
& \psi_{\frac{1}{2} 1}(x ; \lambda)=O\left(\frac{1}{\Im \lambda}\right) \exp \left(b \lambda i\left(x-\frac{1}{2}\right)\right), \\
& \psi_{\frac{1}{2} 2}(x ; \lambda)=O\left(\frac{1}{\Im \lambda}\right) \exp \left(a \lambda i\left(x-\frac{1}{2}\right)\right) .
\end{aligned}
$$

Using the formula (2.56) and the estimates (2.3), (2.4), (2.58) - (2.61) one gets the following estimates for the functions in (2.48)

$$
\vec{\omega}_{i}(x ; \lambda)=O\left(\lambda^{M} \exp (i a \lambda(x+1))\right), \quad \text { if } \Im \lambda>0
$$

and

$$
\vec{\omega}_{i}(x ; \lambda)=O\left(\lambda^{M} \exp (i b \lambda(x+1))\right), \quad \text { if } \Im \lambda<0 .
$$

From the estimates $(2.46),(2.47),(2.62),(2.63)$ one gets finally the estimates

$$
F_{i}(\lambda)=O\left(\frac{1}{\sqrt{|\Im \lambda|}}\right), \quad|\Im \lambda|>C,
$$

where $C>0$ is a constant. Then, by Phragmen-Lindelöf theorem for a strip, one again concludes that $F_{j}(\lambda) \equiv 0$, and therefore $\tilde{F}_{j}(\lambda) \equiv 0$, i.e. $\vec{f}(x)$ is orthogonal to $\vec{\omega}_{1}(x ; \lambda)$ and $\vec{\omega}_{2}(x ; \lambda)$ for all $\lambda$.

Observe, that if $\chi(\lambda) \neq 0$ then the functions $\vec{\omega}_{1}(x ; \lambda)$ and $\vec{\omega}_{2}(x ; \lambda)$ are linearly independent. Therefore, for these values of $\lambda, \vec{\omega}_{1}(x ; \lambda)$ and $\vec{\omega}_{2}(x ; \lambda)$ form a fundamental system of solutions of the system (1.6). Thus, $\vec{f}(x)$ is orthogonal to all solutions of the system (1.6). Consequently, $\vec{f}(x) \equiv 0$ and this completes the proof.

\section{RiESZ BASIS PROPERTY OF THE SEAF}

In this section some sufficient conditions for the Riesz basis property of the SEAF of the system (1.6) with separated $\lambda$-depending boundary conditions will be established.

First recall the definition of the Riesz basis.

Definition 3.1. A system of vectors $\left\{\psi_{n}\right\}_{n=1}^{\infty}$ is called a Riesz basis in the Hilbert space $H$ if there exists a bounded operator $A$ with bounded inverse $A^{-1}$, such that the transformed system $\left\{A \psi_{n}\right\}_{n=1}^{\infty}$ forms an orthonormal basis in $H$.

The following lemma is well known (see [4, Chap. VI, §3]).

Lemma 3.2. Let the system of the vectors $\left\{\psi_{n}\right\}_{n=1}^{\infty}$ be complete in a Hilbert space $H$. Let $\left\{\varphi_{n}\right\}_{n=1}^{\infty}$ be a Riesz basis of $H$ such that $\sum_{n=1}^{\infty}\left\|\psi_{n}-\phi_{n}\right\|^{2}<\infty$. Then the system $\left\{\psi_{n}\right\}_{n=1}^{\infty}$ is a Riesz basis of $H$, too.

Also, the following lemma will be needed, which concerns the spectrum of the system (1.6) with separated $\lambda$-depending boundary conditions.

Lemma 3.3. Let the function $Q(x)$ be differentiable.

Let $P_{11}(\lambda)$ and $P_{12}(\lambda)$ be relatively prime polynomials with $\operatorname{deg} P_{11}=\operatorname{deg} P_{12}=N_{0}$ and let $P_{21}(\lambda)$ and $P_{22}(\lambda)$ be relatively prime polynomials with $\operatorname{deg} P_{21}=\operatorname{deg} P_{22}=N_{1}$.

Let $C_{i j}$ be the leading coefficient of the polynomial $P_{i j}(\lambda)$ and denote $C_{1}=C_{11} C_{21}$ and $C_{2}=C_{12} C_{22}$. 
Let the set $\Lambda$ contain $N=N_{0}+N_{1}$ eigenvalues of the problem (1.6) with separated $\lambda$-depending boundary conditions given by

$$
\left\{\begin{array}{l}
P_{11}(\lambda) y_{1}(0)+P_{12}(\lambda) y_{2}(0)=0 \\
P_{21}(\lambda) y_{1}(1)+P_{22}(\lambda) y_{2}(1)=0
\end{array} .\right.
$$

Then it is possible to enumerate the remaining eigenvalues, so that

$$
\lambda_{n}=\frac{i \ln \left(C_{1} / C_{2}\right)+2 \pi n}{b-a}+O\left(\frac{1}{|n|}\right), \quad \text { where } n \in \mathbb{Z} .
$$

Proof. The characteristic function $\chi(\lambda)$ of the system (1.6) with the boundary conditions in (3.1) has the form

$$
\begin{aligned}
\chi(\lambda)=P_{11}(\lambda)\left(P_{21}(\lambda) \psi_{01}(1 ; \lambda)+P_{22}(\lambda) \psi_{02}(1 ; \lambda)\right) & \\
& -P_{12}(\lambda)\left(P_{21}(\lambda) \varphi_{01}(1 ; \lambda)+P_{22}(\lambda) \varphi_{02}(1 ; \lambda)\right),
\end{aligned}
$$

where the products of polynomials are all of degree $N$ by assumptions.

Introduce the function

$$
\Pi(\lambda)=\prod_{\tilde{\lambda}_{s} \in \Lambda}\left(\lambda-\tilde{\lambda}_{s}\right)^{p_{s}}
$$

where $p_{s}$ is the multiplicity of the eigenvalue $\tilde{\lambda}_{s}$ in the set $\Lambda$.

Then the eigenvalues which do not belong to the set $\Lambda$, are the roots the entire function

$$
\tilde{\chi}(\lambda):=\frac{\chi(\lambda)}{\Pi(\lambda)} .
$$

It follows from Lemma 2.2 that the function $\tilde{\chi}(\lambda)$ satisfies the following estimate:

$$
\tilde{\chi}(\lambda)=C_{1} \exp (a \lambda i)-C_{2} \exp (b \lambda i)+\frac{1}{\lambda} O(\max \{\exp (a \lambda i), \exp (b \lambda i)\}),
$$

where $C_{1} C_{2} \neq 0$.

On the line

$$
\Re \lambda=\frac{\Im \ln \left(C_{1} / C_{2}\right)+(2 n+1) \pi}{b-a},
$$

which is determined by the equation $\arg \left(C_{1} \exp (a \lambda i)\right)=\arg \left(-C_{2} \exp (b \lambda i)\right)$, one has

$$
\left|C_{1} \exp (a \lambda i)-C_{2} \exp (b \lambda i)\right|=\left|C_{1} \exp (a \lambda i)\right|+\left|C_{2} \exp (b \lambda i)\right| \text {. }
$$

From (3.3) and (3.4) one concludes that on this line, with $|\lambda|$ large enough,

$$
\left|\tilde{\chi}(\lambda)-\left(C_{1} \exp (a \lambda i)-C_{2} \exp (b \lambda i)\right)\right|<\left|C_{1} \exp (a \lambda i)-C_{2} \exp (b \lambda i)\right| .
$$

Therefore, it follows from Rouche's theorem (see [16, Theorem 10.43; Exercise 24, p.229]) and the estimates (3.3) and (3.5) that for $|n|$ large enough there exists precisely one root of $\chi(\lambda)$ in the strip

$$
(2 n-1) \pi<(b-a) \Re \lambda+\Im \ln \left(C_{1} / C_{2}\right)<(2 n+1) \pi,
$$

and, furthermore, that there are $2|n|-1$ roots of the function $\tilde{\chi}(\lambda)$ in the strip

$$
-(2|n|-1) \pi<(b-a) \Re \lambda+\Im \ln \left(C_{1} / C_{2}\right)<(2|n|-1) \pi
$$

Therefore the roots of $\tilde{\chi}(\lambda)$ except for, possibly, a finite number of them, are simple. Moreover, the roots $\lambda_{n}$ of $\tilde{\chi}(\lambda)$ can be ordered as a bilateral sequence, so that for $|n|>n_{0}$, $\lambda_{n}$ belongs to the strip (3.6).

Let

$$
\lambda_{n, 0}=\frac{i \ln \left(C_{1} / C_{2}\right)+2 \pi n}{b-a}
$$


be the root of the function

$$
\tilde{\chi}_{0}(\lambda)=C_{1} \exp (a \lambda i)-C_{2} \exp (b \lambda i) .
$$

Consider a disk $D(n, \rho)$ with the radius $\rho$ and the center $\lambda_{n, 0}$. For $\lambda \in D(n, \rho)$ it follows from (3.3) that there exist $K_{1}$, such that

$$
|\lambda|\left|\tilde{\chi}(\lambda)-\tilde{\chi}_{0}(\lambda)\right|<K_{2}(|\exp (a \Im \lambda)|+|\exp (b \Im \lambda)|)<K_{1} .
$$

Moreover, because $\Im \lambda$ is independent of $n, K_{1}$ is independent of $n$, too.

On the other hand, because the derivative of the function $\tilde{\chi}_{0}(\lambda)$ at $\lambda_{n, 0}$ is nonzero, then, for $\rho$ small enough there exists $K_{3}$, such that $\left|\tilde{\chi}_{0}(\lambda)\right|>K_{3}\left|\lambda-\lambda_{0}\right|$.

Therefore, if $\rho \geq \frac{K_{1}}{K_{3}|\lambda|}$ and $\left|\lambda-\lambda_{0}\right|=\rho$, then (3.9) implies that

$$
\left|\tilde{\chi}_{0}(\lambda)\right|>\frac{K_{1}}{|\lambda|}>K_{2} \frac{|\exp (a \Im \lambda)|+|\exp (b \Im \lambda)|}{|\lambda|}>\left|\tilde{\chi}(\lambda)-\tilde{\chi}_{0}(\lambda)\right| .
$$

Hence, again by Rouche's theorem, in this disk the functions $\tilde{\chi}(\lambda)$ and $\tilde{\chi}_{0}(\lambda)$ have the same number of roots, i.e., precisely one root.

Therefore, $\left|\lambda_{n}-\lambda_{n, 0}\right|<\frac{K_{1}}{K_{3}\left|\lambda_{n}\right|}$. Since $\lambda_{n}=\frac{2 \pi n}{b-a}+O(1)$, one has $\frac{K_{1}}{K_{3}\left|\lambda_{n}\right|}=O\left(\frac{1}{|n|}\right)$.

Now, using the formula (3.8), the statement in (3.2) follows.

Lemmas 3.2 and 3.3 are used to prove the following theorem.

Theorem 3.4. Let $P_{11}(\lambda)$ and $P_{12}(\lambda)$ be relatively prime polynomials with $\operatorname{deg} P_{11}=$ $\operatorname{deg} P_{12}=N_{0}$ and let $P_{21}(\lambda)$ and $P_{22}(\lambda)$ be relatively prime polynomials with $\operatorname{deg} P_{21}=$ $\operatorname{deg} P_{22}=N_{1}$.

Let $\Phi$ be a set, which consists of $N=N_{0}+N_{1}$ eigenfunctions and associate functions of the problem (1.6), (3.1) and assume that the SEAF of this problem without the set $\Phi$ is complete in the space $L^{2}[0,1] \oplus L^{2}[0,1]$.

Then the SEAF of problem (1.6), (3.1) without the set $\Phi$ is a Riesz basis in the space $L^{2}[0,1] \oplus L^{2}[0,1]$.

Proof. By Lemma 3.3, it is possible to enumerate the eigenvalues $\lambda_{n}$, corresponding to the eigenfunctions $\overrightarrow{\omega_{n}}(x)$ which are not contained in the set $\Phi$, such that

$$
\lambda_{n}=\frac{i \ln \left(C_{1} / C_{2}\right)+2 \pi n}{b-a}+O\left(\frac{1}{|n|}\right) .
$$

Because $\overrightarrow{\omega_{n}}(x)$ satisfies the first of the conditions in (3.1), it may be written in the form $\overrightarrow{\omega_{n}}(x)=P_{12}\left(\lambda_{n}\right) \overrightarrow{\varphi_{0}}\left(x ; \lambda_{n}\right)-P_{11}\left(\lambda_{n}\right) \overrightarrow{\psi_{0}}\left(x ; \lambda_{n}\right)$ (up to a constant multiplier)

Then, by Lemma 2.2 ,

$$
\overrightarrow{\omega_{n}}(x)=\left(\begin{array}{c}
C_{12} \exp \left(a \lambda_{n} i x\right) \\
-C_{11} \exp \left(b \lambda_{n} i x\right)
\end{array}\right)+\frac{1}{\lambda_{n}}\left(O\left(\exp \left(a \lambda_{n} i x\right)\right)+O\left(\exp \left(b \lambda_{n} i x\right)\right)\right) .
$$

Combining the estimates (3.2) in Lemma 3.3 with (3.11) one obtains

$$
\begin{gathered}
\overrightarrow{\omega_{n}}(x)=\left(\begin{array}{c}
C_{12} \exp \left(a \frac{i \ln \left(C_{1} / C_{2}\right)+2 \pi n}{b-a} i x\right) \\
-C_{11} \exp \left(b \frac{i \ln \left(C_{1} / C_{2}\right)+2 \pi n}{b-a} i x\right)
\end{array}\right)+\frac{1}{n}\left(O\left(\exp \left(a \frac{i \ln \left(C_{1} / C_{2}\right)+2 \pi n}{b-a} i x\right)\right)\right. \\
\left.+O\left(\exp \left(b \frac{i \ln \left(C_{1} / C_{2}\right)+2 \pi n}{b-a} i x\right)\right)\right)=\left(\begin{array}{c}
C_{12} \exp \left(a \frac{i \ln \left(C_{1} / C_{2}\right)+2 \pi n}{b-a} i x\right) \\
-C_{11} \exp \left(b \frac{i \ln \left(C_{1} / C_{2}\right)+2 \pi n}{b-a} i x\right)
\end{array}\right)+O\left(\frac{1}{n}\right) .
\end{gathered}
$$


Now define the operator $A: L_{2}[0,1] \oplus L_{2}[0,1] \rightarrow L_{2}[a, b]$ via

$$
A\left(\begin{array}{l}
y_{1} \\
y_{2}
\end{array}\right)(x)= \begin{cases}\frac{1}{C_{12}} y_{1}\left(\frac{x}{a}\right), & \text { where } a<x<0 \\
-\frac{1}{C_{11}} y_{2}\left(\frac{x}{b}\right), & \text { where } 0<x<b .\end{cases}
$$

Then $A$ and $A^{-1}$ are bounded. Therefore, the system $\overrightarrow{\omega_{n}}(x)$ is a Riesz basis in the space $L_{2}[0,1] \oplus L_{2}[0,1]$ if and only if the system $A\left(\overrightarrow{\omega_{n}}\right)$ is a Riesz basis in the space $L_{2}[a, b]$.

From the estimate (3.12) and the definition of $A$ in (3.13) one obtains

$$
A\left(\overrightarrow{\omega_{n}}\right)=\exp \left(\frac{i \ln \left(C_{1} / C_{2}\right)+2 \pi n}{b-a} i x\right)+O\left(\frac{1}{n}\right) .
$$

It is obvious, that the system

$$
\tilde{\omega}_{n}=\exp \left(\frac{i \ln \left(C_{1} / C_{2}\right)+2 \pi n}{b-a} i x\right)
$$

is an orthogonal basis in the space $L_{2}[a, b]$ and that the norms of $\tilde{\omega}_{n}$ are given by

$$
\left\|\tilde{\omega}_{n}\right\|=\int_{a}^{b} \exp \left(-2 \frac{\Re\left(\ln \left(C_{1} / C_{2}\right)\right)}{b-a} x\right) d x
$$

for all $n$. From the estimate (3.14) one concludes that

$$
\sum_{n=-\infty}^{\infty}\left\|A\left(\overrightarrow{\omega_{n}}\right)-\tilde{\omega}_{n}\right\|^{2}<\infty
$$

Therefore, by Lemma $3.2, A\left(\overrightarrow{\omega_{n}}\right)$ is a Riesz basis in $L_{2}[a, b]$.

From Theorem 3.4 one obtains the following result.

Theorem 3.5. Let $\operatorname{deg} P_{11}=\operatorname{deg} P_{12}=0$, i.e., $P_{11} \neq 0$ and $P_{12} \neq 0$ are constants. Let $P_{21}(\lambda)$ and $P_{22}(\lambda)$ be relatively prime polynomials with $\operatorname{deg} P_{21}=\operatorname{deg} P_{22}=N$.

Let $\Phi$ be a set, which consists of $N$ eigenfunctions and associate functions, which satisfies the following condition:

If $\Phi$ contains either an eigenfunction or an associate function corresponding to an eigenvalue $\lambda_{k}$, then it also contains all the associate functions of higher order corresponding to the same eigenvalue.

Then the SEAF of the problem (1.6), (3.1) without the set $\Phi$ is a Riesz basis in the space $L^{2}[0,1] \oplus L^{2}[0,1]$.

Proof. In this case, by Theorem 2.5, the SEAF of the problem (1.6), (3.1) without the set $\Phi$ is complete in the space $L^{2}[0,1] \oplus L^{2}[0,1]$.

Therefore, by Theorem 3.4 it is a Riesz basis.

By taking $N=0$ in Theorem 3.5 yields the following corollary for the system (1.6) with boundary conditions not depending on a spectral parameter, see [21].

Corollary 3.6. ([21]) Let $h_{1}$ and $h_{2}$ be nonzero numbers. Then the SEAF of problem (1.6) with the boundary conditions

$$
\left\{\begin{array}{l}
y_{1}(0)+h_{1} y_{2}(0)=0 \\
y_{1}(1)+h_{2} y_{2}(1)=0
\end{array}\right.
$$

is a Riesz basis in the space $L^{2}[0,1] \oplus L^{2}[0,1]$. 


\title{
REFERENCES
}

[1] N. I. Akhiezer, I. M. Glazman, Theory of linear operators in Hilbert space, Moskva, "Nauka", 1966.

[2] P. Djakov, B. Mityagin, Instability zones of periodic 1D Schrödinger and Dirac operators (Rus), Uspehi Mat. Nauk 61 No4 (2006) 77-182 (English: Russian Math. Surveys 61 №4 (2006) 663-766).

[3] P. Djakov, B. Mityagin, Bari-Markus property for Riesz projections of Hill operators with singular potentials, Manuscript, arXiv:0803.3170.

[4] I.C. Gohberg, M.G. Krĕ̌n, Introduction to the theory of linear nonselfajoint operators, Moskva, "Nauka", 1965.

[5] S. Hassi, L.L. Oridoroga, Completeness theorems for Dirac-type operators with boundary conditions of general form depending on the spectral parameter (Rus), Math. Notes 74 N-2 (2003) 316-320.

[6] S. Hassi, L.L. Oridoroga, Completeness and Riesz basis property of systems of eigenfunctions and associated functions of Dirac-type operators with boundary conditions depending on the spectral parameter, Math. Notes 79 No-4 (2006) 636-640.

[7] M.V. Keldysh, On the characteristic values and characteristic functions of certain classes of non-selfadjoint equations (Russian), Dokl. Akad. Nauk SSSR 77 No1 (1951) 11-14.

[8] B.M. Levitan, I.S. Sargsjan, Introduction to the spectral theory, Moscow, "Nauka", 1970.

[9] M.M. Malamud, Problems of the uniqueness in the inverse problems for the system of differential equations in bounded interval, Trans. Moscow Math. Soc. 60 (1999) 199-258.

[10] M.M. Malamud, L.L. Oridoroga, Theorems of the completeness for the systems of ordinary differential equations, Functional Analysis and Applications 34 №3 (2000) 88-90.

[11] V.A. Marchenko, Sturm - Liouville operators and their applications, Kyiv, "Naukowa dumka", 1977.

[12] R. Mennicken, M. Möller, Non-self-adjoint boundary eigenvalue problems. North-Holland Mathematics Studies, 192. North-Holland Publishing Co., Amsterdam, 2003.

[13] B. Mityagin, Spectral expansions of one-dimensional periodic Dirac operators, Dyn. Partial Differ. Equ. 1 N-2 (2004) 125-191.

[14] M.A. Nămark, Linear differential operators, Moscow, "Nauka", 1968.

[15] L.L. Oridoroga, Boundary value problems for $2 \times 2$ Dirac type systems with spectral parameter in boundary conditions, Methods of Functional Analysis and Topology 7 N-1 (2001) 82-87.

[16] W. Rudin, Real and complex analysis (Third Edition), McGraw-Hill, 1986.

[17] A.A. Shkalikov, The completeness of eigenfunctions and associated functions of an ordinary differential operator with irregular-spliting boundary conditions, Functional Analysis and Applications 10 N-4 (1976) 69-80.

[18] E.I. Tarapowa, Boundary-value problem of Sturm - Liouville equations with nonlinear boundary conditions. I, Theory of Functions, Functional Analysis and Applications 31 (1979) 157-160.

[19] E.I. Tarapowa, Boundary-value problem of Sturm - Liouville equations with nonlinear boundary conditions. II, Theory of Functions, Functional Analysis and Applications 33 (1979) 82-87.

[20] C. Tretter, Spectral problems for systems of differential equations $y^{\prime}+A_{0} y=\lambda A_{1} y$ with $\lambda$-polynomial boundary conditions, Math. Nachr. 214 (2000), 129-172.

[21] I. Trooshin, M. Yamamoto, Riesz basis of root vectors of a nonsymmetric system of first-order ordinary differential operators and application to inverse eigenvalue problems, Appl. Anal. 80 No-1-2 (2001) 19-51.

[22] I. Trooshin, M. Yamamoto, Spectral properties and an inverse eigenvalue problem for nonsymmetric systems of ordinary differential operators, J. Inverse Ill-Posed Probl. 10 N-6 (2002) 643-658.

[23] B.L. van der Waerden, Algebra, Frederick Ungar Publishing Co., New York, 1970.

\author{
Department of Mathematics and Statistics, University of VaAsa, P.O. Box 700, 65101 \\ VAASA, FinLAND
}

Department of Mathematical Analysis, Donetsk National University, UniversitetSKAYA STR. 24, 83055 DONETSK, UKRAINE 